\title{
Atrial myxoma: a treatable cause of stroke
}

\author{
JOSEPH C. MAROON AND ROBERT L. CAMPBELL \\ From the Department of Surgery, Division of Neurological Surgery, Indiana University Medical Center, \\ Indianapolis, Indiana, U.S.A.
}

In the search for treatable causes of cerebrovascular occlusion, little emphasis has been given to emboli from left atrial myxomas. Although rare-only 350 cases had been reported by 1965 (Newman, Cordell, and Prichard, 1966) - it is a histologically benign growth that is completely curable if promptly recognized and surgically removed. From various reviews of the clinical manifestations of this neoplasm (Aldridge and Greenwood, 1960; Goodwin, 1963; Silverman, Olwin, and Graettinger, 1962), systemic embolization is known to occur in 40 to $45 \%$ of patients. Emboli are frequently multiple and lodge in many sites, but in approximately one-half of the cases the brain is the target organ (Silverman et al., 1962). Joynt, Zimmerman, and Khalifeh (1965) recently analysed 12 case reports of patients with left atrial myxoma in whom acute neurological disturbances-usually hemiplegia, unconsciousness, or both-were the presenting clinical features. Although the average duration of neurological symptoms was over one year, all 12 patients died and in only one case was the correct diagnosis made before death. The present report describes a patient who initially presented as a neurological problem and in whom a left atrial myxoma was correctly diagnosed and successfully treated. Three similar cases are summarized to emphasize the subtle but pertinent clinical features which should suggest this diagnosis in the 'stroke' patient.

\section{PRESENT CASE}

CASE 1 A 34-year-old white female was admitted to the Indiana University Medical Center on 19 February 1968 because of recurrent attacks of dysphasia and right hemiparesis. Before 5 August 1964 she was in good health and had borne six children without difficulty. At that time, without prodromal symptoms, she suddenly lost consciousness and remained comatose for five days. Upon wakening, she was dysphasic and had a profound right hemiparesis. No cardiac abnormalities were recorded, a left carotid arteriogram was interpreted as normal, and she was not diabetic. After intensive physical therapy, speech and strength improved and she returned to her previous household duties.

In December 1965 she had a sudden syncopal attack which lasted three hours. She awoke to find that she was again markedly dysphasic and severely weak in her right limbs. During the next several months she improved but required a leg brace and cane for walking. In January 1967, again without warning, she 'blacked-out' for five hours and suffered an exacerbation in her dysphasia and right-sided weakness. She was treated at home and was not further studied because the recurrent attacks were thought to represent some untreatable form of cerebrovascular thrombosis. Distressed by persistent physical disability and fearful of a recurrent attack, she sought other medical advice and she was referred to the neurosurgical service for evaluation of possible carotid artery disease.

Past medical history revealed that, at the age of 18 , during a routine physical examination, she was told that she had an insignificant heart murmur. This finding was never recorded on later physical examinations. She was aware of an occasional irregularity in her pulse, but this rarely persisted for more than a few minutes. There was no history of cardiac decompensation, except for mild dyspnoea on exertion which she first experienced three weeks before hospital admission.

On examination she was a well-developed, wellnourished woman who appeared older than her 34 years. The blood pressure was $135 / 75 \mathrm{~mm} \mathrm{Hg}$. The radial pulse was 86 per minute with occasional irregularities. The cardiac apex beat was at the fifth intercostal space in the mid-clavicular line. There was a palpable first heart sound with no thrusts or thrills. The first heart sound was split and a Grade 2 late systolic blowing murmur was heard at the apex and radiated into the axilla. The second sound was normal. Occasionally, a distinct opening 'snap' followed by a diastolic 'rumble' was heard. This murmur was best appreciated following a premature beat and with the patient in the left lateral decubitus position. On neurological examination she had a slight expressive dysphasia, a spastic right hemiparesis with hyperactive reflexes, ankle clonus, and an extensor plantar response on the right. From the history and physical findings, the initial diagnosis was a left atrial myxoma with multiple cerebral emboli.

The following laboratory studies were performed: haemoglobin 7.2 g/100 ml., WBC $9,600 / \mathrm{cu}$. mm, sedimentation rate $26 \mathrm{~mm} / \mathrm{hr}$, reticulocyte count $1.7 \%$, bilirubin $1.5 \mathrm{mg} / 100 \mathrm{ml}$., serum iron binding capacity $9 \mu \mathrm{g} / 100 \mathrm{ml}$., total iron binding capacity $245 \mu \mathrm{g} / 100 \mathrm{ml}$., direct and indirect Coombs test negative, serum protein electrophoresis showed increase in all gamma globulins 
and decrease in albumins. The serum phosphorus, calcium, alkaline phosphatase, and the SGOT electrolytes and prothrombin time were normal. Blood cultures on four occasions were negative. The electrocardiogram, chest radiograph, and cardiac fluoroscopy were normal. A radiographic bone survey demonstrated radiolucent areas in the left humerus and the right iliac crest. A biopsy of the right iliac crest showed histological changes consistent with bone infarction, but no tumour cells were found. Cineangiocardiography demonstrated a large irregular mass in the left atrium which prolapsed through the mitral valve during diastole.

On 6 April 1968 the patient's left atrium was explored under cardiopulmonary bypass. A mass which measured $6 \mathrm{~cm}$ in length and had a base of $1 \frac{1}{2} \mathrm{~cm}$ attached to the interatrial septum near the fossa ovalis was completely removed without complications. The tumour weighed $56 \mathrm{~g}$ and was of a gelatinous nature. The surface was quite nodular (Fig. 1) and was covered by thrombus. Microscopic examination revealed the characteristic loose oedematous stroma and endothelial proliferation of an atrial myxoma. The post-operative course was uncomplicated. Intensive physical rehabilitation was initiated, and there was a progressive improvement in her neurological condition as well as in her mental state. She was discharged three weeks after surgery.

\section{SUMMARY OF CASES REPORTED PREVIOUSLY}

CASE 2 (Thompson, Rabin, Sosa, and Cronin, 1966) A 29-year-old man first experienced the onset of intermittent paraesthesias in the left upper extremity during the winter of 1961. In March 1962 he suddenly developed slurred speech, a staggering gait and paraesthesias in the left arm, left leg, and right leg. He was then hospitalized for the first time and found to have a left hemiparesis with associated left hyper-reflexia and a left extensor plantar response. The cardiac examination, including an electrocardiogram, was entirely normal. The white blood count was 13,000/cu. $\mathrm{mm}$ and the haemoglobin and erythrocyte sedimentation rate were normal. Because of persistent proteinuria, haematuria, and an increased calcium excretion, a renal biopsy was performed. This demonstrated changes compatible with focal embolic glomerulonephritis. He was discharged with no specific diagnosis.

In August 1962 he developed painful red spots on the hands and feet, and the diagnosis was made of 'purpuric and telangiectatic lesions of a thromboembolic nature, secondary to a systemic disease'. Over the next four months he developed a right upper monoparesis, right facial weakness, and on one occasion was unable to speak for one hour. A parathyroidectomy was performed because of the persistent hypercalcaemia, but this had no effect on his neurological condition.

On 1 January 1963 he suddenly developed dysarthria, ataxia, and a right hemiparesis and was admitted to the hospital on the neurological service. A diagnosis of left atrial myxoma was made on the basis of recurrent peripheral embolization in the presence of a normal cardiac rhythm. This diagnosis was confirmed by cineangiocardiography. On 29 March 1963 a friable left atrial myxoma was removed without complications under total cardiopulmonary bypass. Twenty-three months after surgery he still had a slight residuat neurological deficit but this did not prevent him fror returning to his former employment.

CASE 3 (Stoane, Allen, and Collins, 1966) A 29-year-ol man, over the course of a few months, developed pain and tenderness in the legs and minute haemorrhagic spote on the palms of both hands and under the nail beds. He was hospitalized in May 1963 and was found to have splinter haemorrhages in the nail beds, an absent right dorsalis pedis pulse, and tender calves bilaterally. The cardiac examination was normal, although an electro-

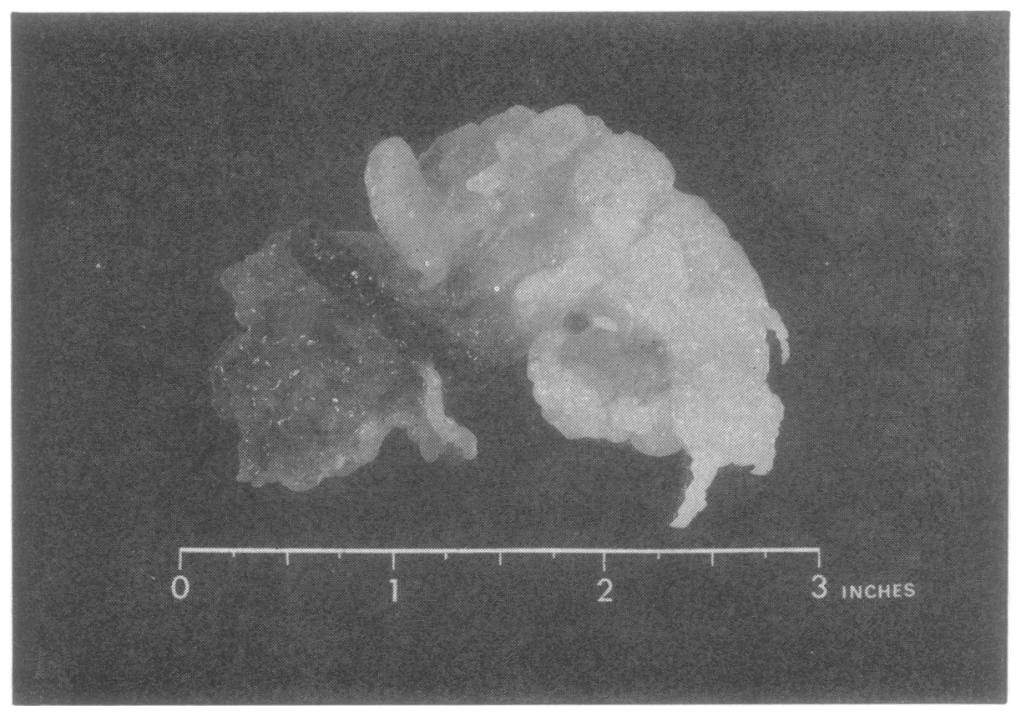

FIG. 1. Excised portion of left atrial wall with attached myxoma. Note the characteristic gelatinous, semi-translucent appearance of the neoplasm. 
cardiogram was suggestive of left ventricular hypertrophy. No specific diagnosis was made.

In November 1963 he developed a sudden left hemiparesis and was diagnosed as having had a 'stroke'. There was a partial recovery after treatment with anticoagulants, and he was able to return to his work.

Approximately one year later he was found unconscious and was immediately hospitalized. On examination the right pupil was larger than the left and optic atrophy was observed in the right eye. There was a moderate right hemiparesis with bilateral hyperactive reflexes. A Grade 1 early systolic murmur was the only abnormal cardiac finding. Erythematous maculopapular 'spots' were seen on the palms of both hands, and a biopsy of one of these revealed a myxomatous thrombus in a small artery with some perivascular inflammation. Bilateral carotid angiograms demonstrated partial occlusion of the left middle cerebral artery and 'pseudoaneurysms' on several branches of the right middle cerebral artery. Cineangiocardiography clearly outlined a left atrial tumour. This was excised without complications under total cardiopulmonary bypass. There was little improvement in the patient's post-operative neurological condition.

CASE 4 (Stoane et al., 1966) A 13-year-old girl first developed the sudden onset of confusion, ataxia, and dysarthria, all of which lasted approximately one and a half hours, in May 1960. Fourteen months later because of a similar episode she was hospitalized. On examination, she had a right supranuclear facial palsy, weakness of the right arm, and increased reflexes on the right. There was marked pallor of the left fundus. The only other abnormal finding was a Grade 2 short systolic apical murmur. Laboratory studies, including a lumbar puncture, were normal and no diagnosis was made. Over the next four months, two additional syncopal episodes occurred which were associated with transient left-sided weakness and incoherence.

There were no further attacks until November 1964. At that time she developed suddenly left-sided paraesthesias, a marked left hemiparesis, and became incontinent. The neurological findings were confirmed on hospital admission, and the short systolic cardiac murmur was again noted. A right carotid angiogram demonstrated partial occlusion of the external carotid artery and a local interruption of flow in the middle cerebral artery. Multiple small filling defects were seen in the pericallosal branches. Cineangiocardiography demonstrated a large pedunculated mass located primarily in the left ventricle. This proved to be a large gelatinous atrial myxoma which was removed without complications under cardiopulmonary bypass. Post-operatively there was a progressive improvement in her neurological condition.

\section{DISCUSSION}

Recently, there have been several excellent reviews of the clinical manifestations of left atrial myxoma (Goodwin, 1968). In general, symptoms are produced by obstruction to cardiac blood flow by a large or critically placed tumour, through constitutional effects, or by embolization. Blood flow obstruction, with the concomitant development of congestive heart failure refractory to the usual cardiac regimen and the production of various cardiac murmurs, is the most frequent clinical presentation. Goodwin, Stanfield, Steiner, Bentall, Sayed, Bloom, and Bishop (1962) have emphasized the diagnostic importance of the constitutional symptoms of fever, malaise, weight loss, and various haematological alterations in patients with cardiac myxomas. From the present case and others (Krapin, 1963; Joynt et al., 1965), however, it is seen that neurological symptoms and even death may occur from cerebral embolization before any obstructive or constitutional effects are manifest.

It is well known that most cerebral emboli originate from an atrial thrombus in a fibrillating heart, a mural thrombus from the site of a previous myocardial infarction, or from calcified or infected mitral valves. In the patient with suspected cerebral embolus, however, there are several features which should at least suggest the possibility of a left atrial tumour.

AGE Myxomas may occur at any age. They have been found in the newborn (Reddy, Suryaprakasa Rao, Venkaiah, Gopalakrishnaiah Gupta, Sakuntala Devi, and Venkataswamy Naidu, 1956) as well as in those over 90 (Hudson, 1965), but they are most commonly diagnosed in patients between the third and sixth decade. A feature receiving little emphasis in previous reports is the significance of cerebral embolization from cardiac tumours in young patients. Three of the patients described above experienced major neurological symptoms secondary to embolic episodes before the age of 30 ; moreover, one summarized here (Stoane et al., 1966) and two patients reported by Joynt et al. (1965) first developed neurological symptoms at the ages of 13,9 , and 13 respectively. Because of the rarity of cerebral embolization in those under 30 , it is suggested that, after excluding bacterial endocarditis, all patients in this age group with findings of acute cerebrovascular occlusion should be considered for cineangiocardiography.

CARDIAC SIGNS There are no cardiac signs specific for left atrial tumours. A normal heart rhythm, however, is commonly associated with atrial myxomas (Aldridge and Greenwood, 1960), and suspicion should be aroused whenever cerebral embolism occurs in a patient with a non-fibrillating heart. A more significant but less commonly recorded finding is a cardiac arrhythmia which is related to changes in body position. This was observed in case 1 and was of definite diagnostic significance. 
Left atrial tumours commonly produce heart murmurs by interfering with mitral valve function. A diastolic murmur characteristic of mitral stenosis, a systolic murmur of mitral insufficiency, or both, with or without an opening snap may be present. But so variable are the cardiac murmurs that Goodwin (1963), after reviewing 45 cases, concluded that in the typical patient the heart murmurs were atypical or at least difficult to detect. Indeed, heart murmurs were not a prominent feature initially in any of the patients in this series. The finding that was helpful in case 1 was the variation of the murmur with time and with the position of the patient. Since these tumours frequently have relatively long pedicles, depending on body position they are free to prolapse into the left ventricle, temporarily interfere with mitral valve closure, and thus produce variable cardiac murmurs. This variability is often emphasized in case reports as one of the clues leading to a diagnosis of left atrial myxoma.

MULTIPLE SYSTEMIC EMBOLI This in itself is certainly not of particular significance. There are, however, several reports of left atrial myxoma diagnosed only after histological examination of an excised embolus (Beanlands, Roy, Dolan, and Shane, 1960; Zuidema, Burke, Villegas, and Scannell, 1961; Willman, Symbas, Mamiya, Cooper, and Hanlon, 1965). The patient in case 3 had two successive 'strokes' of undetermined cause in the course of a year before a biopsy of recurrent 'red spots', present on the fingers of both hands, demonstrated a myxomatous thrombus in a small artery. Only then was the correct diagnosis made. A biopsy of the right iliac crest in case 1 and the kidney in case 2, both probable targets of emboli, were not diagnostic. It is emphasized, however, that an excised embolus from any location should always be examined histologically, since myxomas have been diagnosed from tumour emboli removed from the spleen, kidney, gastrointestinal tract, bone, digital arteries, skin, aorta, and other large vessels.

CEREBRAL ANGIOGRAPHY In the only report dealing with the cerebral arteriographic features of emboli from left atrial myxoma, Stoane and co-workers (1966) described two patterns: (1) filling defects of varying size, with or without interruption of flow, and (2) local changes in the arterial walls ranging from irregularity and slight dilatation to pseudoaneurysm formation. Neither of these changes were found in the angiograms obtained in case 1. Although they cannot be considered specific for cardiac tumours, as emphasized by Stoane et al., if these angiographic abnormalities are found a cardiac tumour should be considered.
LABORATORY STUDIES An abnormal albumin- $\frac{}{z}$ globulin ratio with hyper-globulinaemia, an elevated sedimentation rate, anaemia, and leukocytosis may ō all be associated with a left atrial myxoma (Goodwin et al., 1962). The anaemia may be non-specific or,,$\hat{a}$ as in case 1 , may be associated with a marked iron ${ }_{0}$ deficiency. Whether this is due to toxic depression of bone marrow, haemolysis secondary to the mech- $\frac{2}{0}$. anical effect of the tumour, or both, is not clear. $\vec{z}$ Since all of these abnormalities are usually associated with symptoms of a generalized systemic disorder, $\stackrel{\vec{\rho}}{\rightarrow}$ Goodwin et al. (1962) have postulated that myxomas 0 may release some abnormal substance which results 듬 in a 'general poisoning of the body tissues', but this $\frac{\bar{D}}{\frac{\bar{T}}{T}}$ has not yet been confirmed.

The most frequent diagnostic pitfall in a patient with a myxoma presenting with the above haemato- क logical disturbances, constitutional symptoms, and $\vec{\circ}$ a sudden neurological deficit is bacterial endocarditis. $\overrightarrow{\vec{\omega}}$ Negative blood cultures, the absence of other $\stackrel{\omega}{\sigma}$ valvular lesions, failure to respond to antibiotics, $\overline{\bar{z}}$ and the absence of splenomegaly may help in making the correct diagnosis.

\section{SUMMARY}

A case with multiple cerebral emboli from a le atrial myxoma which was correctly diagnosed and treated is reported. Three similar cases are suns marized. All patients had acute neurologica abnormalities as the primary problem, and cardis symptoms and signs were minimal or absent.

The following clinical features in a patient with a suspected cerebral embolus should alert the clinician to the possibility of a left heart tumour: (1) a patient under 30 in whom the diagnosis of bacterial endocarditis has been eliminated; (2) the finding of a $\stackrel{\mathbb{\perp}}{\complement}$ normal cardiac rhythm in a patient with no history of $\overrightarrow{\vec{B}}$ previous heart disease; (3) a cardiac arrhythmia $\frac{3}{3}$ and/or murmur which varies with time and body position; (4) hyperglobulinaemia, elevated sedimentation rate, anaemia, and leukocytosis in the presence of constitutional symptoms suggesting a generalized systemic disorder; (5) the discovery of myxomatous tissue in an excised embolus from any 3 organ or vessel; (6) the finding of a filling defect or a pseudoaneurysm on cerebral arteriograms.

\section{REFERENCES}

Aldridge, H. E., and Greenwood, W. F. (1960). Myxoma of the left atrium. Brit. Heart J., 22, 189-200.

Beanlands, D. S., Roy, D. L., Dolan, F. G., and Shane, S. J. (1960). ऽ Myxoma of the left atrium. Canad. med. Ass. J., 83, 715-717. N

Goodwin, J. F. (1963). Diagnosis of left atrial myxoma. Lancet, 1, O 464-468.

_-, Guest Editor. (1968). Symposium on cardiac tumours. Amer. J. Cardiol., 21, 307-376. 
_ Stanfield, C. A., Steiner, R. E., Bentall, H. H., Sayed, H. M., Bloom, V. R., and Bishop, M. B. (1962). Clinical features of left atrial myxoma. Thorax, 17, 91-110.

Hudson, R. E. B. (1965). Cardiovascular Pathology. Edward Arnold, London.

Joynt, R. J., Zimmerman, G., and Khalifeh, R. (1965). Cerebral emboli from cardiac tumors. Arch. Neurol. (Chic.), 12, 84-91.

Krapin, D. (1963). Myxoma of left auricle presenting signs of cerebral lesion. N.Y. St.J. Med., 63, 3136-3138.

Newman, H. A., Cordell, A. R., and Prichard, R. W. (1966). intracardiac myxomas: literature review and report of six cases, one successfully treated. Amer. Surg., 32, 219-230.

Reddy, D. J., Suryaprakasa Rao, T., Venkaiah K. R., Gopalakrishnaiah Gupta, K., Sakuntala Devi, P., and Venkataswamy Naidu, N. (1956). Congenital myxoma of the heart. Indian J. Pediat., 23, 210-212.
Silverman, J., Olwin, J. S., and Graettinger, J. S. (1962). Cardiac myxomas with systemic embolization. Circulation, 26, 99-103.

Stoane, L., Allen, J. R., and Collins, H. A. (1966). Radiologic observations in cerebral embolization from left heart myxomas. Radiology, 87, 262-266.

Thompson, W. G., Rabin, E. Z., Sosa, J. A., and Cronin, R. F. P. (1966). Left atrial myxoma with multiple emboli and hypercalcemia. Canad. med. Ass. J., 95, 316-318.

Willman, V. L., Symbas, P. W., Mamiya, R. T., Cooper, T., and Hanlon, C. R. (1965). Unusual aspects of intracavitary tumors of the heart. Report of two cases. Dis Chest, 47, 669-671.

Zuidema, G. D., Burke, J. F., Villegas, A. H., and Scannell, J. G. (1961). Surgery of atrial myxoma. New Engl.J. Med., 264, 1016. 1021

\section{The February 1969 Issue}

\section{THE FEBRUARY 1969 ISSUE CONTAINS THE FOLLOWING PAPERS}

Motor neurone disease: an abnormality of nerve metabolism BHAGWAN SHAHANI and W. RITCHIE RUSSELL

Electromyographic recording of the jaw reflex in multiple sclerosis C. J. GOODWILL and L. O'TUAMA

Pseudo-myotonia and myokymia R. C. HUGHES and W. B. MATTHEWS

The habituation of forearm muscle blood flow in phobic subjects HARRY BRIERLEY

H reflex studies in patients with cerebellar disorders J. G. MCLEOD

Orthostatic hypotension and nicotine sensitivity in a case of multiple system atrophy J. G. GRAHAM and D. R. OPPENHIEMER

Migraine and multiple sclerosis SYLVIA M. WATKINS and MICHAEL ESPIR
Destruction of peripheral white matter of the spinal cord, brain-stem, and optic tracts REINHARD L. FRIEDE and UROS ROESSMANN

A case of neurofibromatosis complicated by medulloblastoma, neurogenic sarcoma and radiation-induced carcinoma of thyroid A. G. L. CORKILL and C. F. ROSS

Stereotactic treatment of acromegaly LOUIS W. CONWAY, FEARGHUS T. O'FOGHLUDHA, and WILLIAM F. COLLINS

A simple visual alignment device for use with the Leksell stereotactic co-ordinate frame B. H. DAWSON, E. DERVIN, and 0 . B. HEYWOOD

Proceedings of the Society of British Neurological Surgeons: 78th Meeting

Book Reviews

Copies are still available and may be obtained from the PUBLISHING MANAGER, BRITISH MEDICAL ASSOCIATION, TAVISTOCK SQUARE, W.C.1., price 21s. 